\title{
Monitoring the Injured Brain
}

\author{
Chunyan $\mathrm{Li}^{1,2,3}$ and Raj K Narayan ${ }^{1,2,3}$ \\ ${ }^{1}$ Cushing Neuroscience Institute; ${ }^{2}$ The Feinstein Institute for Medical Research; and ${ }^{3}$ Hofstra North Shore-LIJ School of Medicine, \\ Manhasset, New York, United States of America
}

\begin{abstract}
Traumatic brain injury is a serious public health problem in the United States, accounting for nearly 1.7 million injuries and 52,000 deaths annually. The initial brain injury is made worse by secondary events which include, but are not limited to, ischemia, swelling, cell damage and brain functions abnormalities which, while posing therapeutic challenges, offer therapeutic opportunities. Unfortunately, in spite of significant efforts, no effective indisputable treatment which would effectively alleviate consequences of this often devastating event currently exists. Multimodality neuromonitoring could provide early warning of secondary brain injury and guide individualized therapy. However, it is rarely done due to the complexity of using multiple devices and the increased risk of complications. This article presents a novel multifunctional smart catheter to continuously and accurately monitor multiple physiological, metabolic and electrophysiological parameters that are vitally important in guiding the care of patients with traumatic brain injury. In addition to measuring various crucial parameters, the developed smart catheter allows for drainage of excess cerebrospinal fluid as a therapeutic strategy to reduce intracranial pressure. The studies which were performed under a rat permanent middle cerebral artery occlusion model indicate that the smart catheter, a single probe, can dynamically detect changes in cerebral glucose, lactate, oxygen, glutamate, temperature, local cerebral blood flow and intracranial pressure that correlated with spreading depression. These results demonstrate that the smart catheter is capable of simultaneous and continuous measurement of multiple brain variables, within the pathophysiology ranges observed in brain injury. The smart catheter has the potential to improve our understanding of brain pathophysiology and advance the field of neuromonitoring into a completely new era in which medical decisions will be based on comprehensive, real-time measures of brain chemistry and physiology during the critical period immediately following a brain injury.
\end{abstract}

Online address: www.bioelecmed.org

doi: 10.15424/bioelectronmed.2014.00010

\section{INTRODUCTION}

Traumatic brain injury (TBI) is defined as "an alteration in brain function, or other evidence of brain pathology, caused by an external force" (1). It is a major cause of death and disability in both civilian and military settings, with large direct and indirect costs to society. The mortality from severe TBI is approximately $30 \%$ with a substantial proportion of survivors being left with significant disability (2). The initial brain injury is made worse by secondary events which include, but are not limited to, ischemia, swelling, cell damage and brain functions abnormalities (3-5) which, while posing therapeutic challenges, offer therapeutic opportunities. Pharmacological neuroprotective agents have so far failed to demonstrate a statistically significant effect when given after TBI (6). Therefore, current patient management strategies are directed toward optimizing the physiological environment of the injured tissue to minimize secondary insults (7-8). Multimodality monitoring, that is currently only partly available with the use of multiple devices, could provide a

Address correspondence to Chunyan Li, The Feinstein Institute for Medical Research, 350 Community Drive, Manhasset, NY 11030. Phone: 516-562-1078; Fax: 516-562-3030; E-mail: Cli11@NSHS.edu.

Submitted November 10, 2014; Accepted for publication November 10, 2014; Published Online (www.bioelecmed.org) December 2, 2014.

\section{The Feinstein Institute for Medical Research Empowering Imagination. Pioneering Discovery.}

comprehensive assessment of the injured brain and hence enable immediate detection and prevention of irreversible neurologic injury (9-11). Such individualized treatment, informed by multimodality monitoring, has the potential to improve patient outcomes after TBI.

The current approach for multimodality monitoring patients with any type of acute severe brain injury is based on the insertion of multiple probes into the brain parenchyma (12-15). However, it is rarely done due to the complexity of using multiple devices and the increased risk of complications. Hence, even the available monitors are not always used. Monitoring of the injured brain has advanced little in recent decades and is limited mainly to intracranial pressure (ICP) and, less frequently, brain tissue oxygenation. This leads to suboptimal care based mainly on computerized tomography (CT) scans and observation of neurologic status, both of which are intermittent and usually indicate complete 


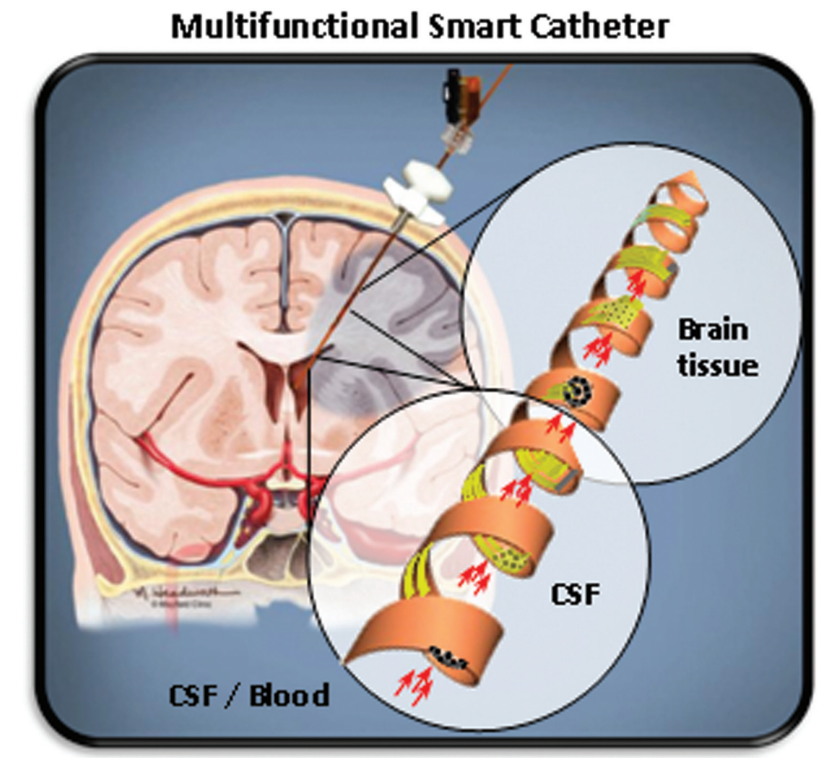

Figure 1. Conceptual drawing of a smart catheter for multimodality monitoring in traumatic brain injury: It can continuously and accurately monitor multiple physiological, metabolic and electrophysiological parameters in the injured brain. Microsensors outside the catheter monitor local brain tissue parameters and the ones outside monitor global cerebrospinal fluid (CSF) parameters. It can also work as a catheter to drain excess CSF.

damage. Part of the reason for this limitation is the lack of a simple, comprehensive and inexpensive monitoring device. Thus, there is a vital need for a single, accurate, multimodality device that can continuously monitor the key biochemical and physiological parameters in severely injured or ill patients and provide us with an "early warning system."

Recent progress in MicroElectroMechanical systems (MEMS) and nanotechnology permits the development of highly functional neural probes for diagnosis and treatment of brain diseases (16-18). The highly integrated probes can be made small and flexible, rendering their usage minimally invasive. The microsensors on the neural probe can achieve better spatial and temporal resolution with high sensitivity and selectivity, a feature highly desired in neuromonitoring applications. Hence, the adoption of MEMS technology has the potential to revolutionize brain multimodality monitoring by miniaturizing and integrating all the desired functions such as ICP monitor, brain oxygen tension monitor, cerebral microdialysis ana- lyzer, and so on, into a single compact unit.

In this paper, we present a novel multifunctional smart catheter developed by multidisciplinary technologies to continuously and accurately monitor multiple physiological, metabolic and electrophysiological parameters that are critically important in guiding the care of patients with TBI (Figure 1). One of the unique aspects of the smart catheter is that it employs a novel packaging technique to assemble multiple microsensors on both the inside and outside of the flexible polymer tube while avoiding wiring and assembling problems associated with previous methods. In addition, the smart catheter allows for drainage of excess cerebrospinal fluid as a therapeutic strategy to reduce ICP. Therefore, this serves both as a monitoring and therapeutic device (19).

\section{METHODS}

Eight microsensors were first fabricated on the flexible polyimide (PI2611) substrate (Total thickness: $18 \mu \mathrm{m}$ thick) based on MEMS and nanotechnology. A novel spiral rolling technique $(20,21)$ was employed to form a flexible polymer tube without wiring and assembling problems associated with conventional approaches. The developed smart catheter exhibits an inner diameter of 0.7 $\mathrm{mm}$, an outer diameter of $0.8 \mathrm{~mm}$ and a sensing length of $5 \mathrm{~mm}$. Our technique allows the microsensors to be located either at inner or outer wall of the tube. The mechanical design and electrical operation of the microsensors were carefully chosen and tailored such that the potential electronic, thermal and chemical cross-talks among the microsensors were minimized. The nonideal crosstalks addressed during the course of sensor miniaturization and integration include electromagnetic interference (EMI); electrical feedthrough across conductive electrodes; chemical crosstalks among the electrochemical sensors; inductive coupling among the traces; finite traces resistance; and capacitive coupling.

To achieve the highest microsensor performances, several nano-/micro-fabrication techniques were developed for the smart catheter: 1) Silicon Nitride film (thickness: $80 \mathrm{~nm}$ ) was directly sputtered on the PI2611 film to provide a better diffusion barrier against humidity (22), one of the major problems for flexible polymer MEMS devices; 2) Nanocrystalline polysilicon was developed by an aluminum-induced crystallization process to realize a highly sensitive pressure sensor on the flexible substrate (23); 3) Platinum nanoparticles were electroplated on the working electrode of the biosensors to achieve higher sensitivity by providing a large available surface and enhancing the electrocatalytic activity $(24)$; 4) Iridium oxide nanoparticles were electroplated on the electroencephalography (EEG) electrodes to achieve a superior signal-to-noise ratio (SNR) and lower polarization rate (25); 5) Calcium peroxide and cerium oxide nanoparticles were integrated into the biosensor enzyme matrix to accurately measure the biochemical signals even under extreme hypoxia (26). 
New structures and operating methods for the microsensors were developed to achieve high accuracy and reliability without cross-talk or interferences among the microsensors: 1) Temperature and cerebral blood flow microsensors were based on micromachined gold resistance temperature detectors (RTD) with a four-wire configuration (27). The temperature sensor operated with pulse excitation current without causing selfheating and its resistance measured by delta method cancelling the thermal electric effect (28); 2) The flow sensor employed a periodic heating and cooling technique with a constant-temperature mode to achieve the highest accuracy (29); 3) An oxygen electrochemical microsensor with three-electrode configuration was designed to achieve zero net oxygen consumption (24); 4) 3D ring-recessed microelectrodes were designed for the electrochemical biosensors to prevent both chemical and electrical cross-talks (30); 5) The temperature sensor was located outside the "thermal influence" area from flow sensor to prevent thermal cross-talk (29).

We have developed the multimodality monitor for the smart catheter. It is comprised of four units. First is the headstage, which performs signal amplification as well as provides mechanical interconnection between the smart catheter and the remote monitor. Second is the mixed signal front-end unit, which performs driving, signal conditioning and data acquisition. The processed outputs are then passed to the digital signal processing unit, which provides functions including data fusion, digital filtering, calibration, display control and recording. The final unit is the display, which contains all the visualization and provides human-machine interface. The multimodality monitor has the following features: 1) Display a total of 11 sensor channels including ICP, temperature, $\mathrm{CBF}$, oxygen, glucose, lactate, glutamate and four full-band ECoG channels; 2) Perform on-demand data trend function; 3) Set the trigger level for the warning alarm as desired; 4) Transfer the recorded

Table 1. Key features for the developed smart catheter microsensors.

\begin{tabular}{llccl}
\hline Parameter & \multicolumn{1}{c}{ Working principle } & Resolution & Accuracy & \multicolumn{1}{c}{ Range } \\
\hline Pressure & $\begin{array}{c}\text { Polysilicon diaphragm } \\
\text { based }\end{array}$ & 0.1 & 1 & $0-50 \mathrm{mmHg}$ \\
Temperature & $\begin{array}{c}\text { Resistance temperature } \\
\text { detector }\end{array}$ & 0.03 & 0.1 & $25-45^{\circ} \mathrm{C}$ \\
Flow & $\begin{array}{c}\text { Thermal diffusion }- \\
\text { periodic heating }\end{array}$ & 0.25 & 5 & $0-200 \mathrm{~mL} / 100 \mathrm{~g}-\mathrm{min}$ \\
Oxygen & $\begin{array}{c}\text { Electrochemical } \\
\text { Glucose }\end{array}$ & 0.2 & 1 & $0-160 \mathrm{mmHg}$ \\
Lactate & $\begin{array}{c}\text { Electrochemical } \\
\text { Electrochemical }\end{array}$ & 0.01 & 0.05 & $0.03-10 \mathrm{mmol} / \mathrm{L}$ \\
ECoG & $\begin{array}{l}\text { Electrochemical } \\
\text { Depth electrode }\end{array}$ & 0.006 & 0.1 & $0.02-8 \mathrm{mmol} / \mathrm{L}$ \\
\hline
\end{tabular}

data easily to the USB flash drive; and 5) Reveal real-time interactions among user-selected sensor channels.

The in vivo application and performance of the smart catheter were tested in the permanent middle cerebral artery occlusion (MCAO) rat model. Experiments involving animals were approved by the Institutional Animal Care and Use Committee (IACUC) of the Feinstein Institute for Medical Research. Male Sprague Dawley rats weighing 250-300 gram ( $\mathrm{n}=$ 6) were used as the subjects. Standard stereotaxic surgical techniques were used to unilaterally implant a precalibrated smart catheter into the neocortex using the following coordinates relative to bregma (AP: $-1.5 \mathrm{~mm}, \mathrm{ML}: 5.0 \mathrm{~mm}$, DV: $-5.0 \mathrm{~mm}$ ). According to previous studies, the lateral location is equivalent to the penumbra of the ischemic area (31). Two small screws were inserted into the skull to anchor the dental cement for securing the smart catheter. Once the dental cement was dried, the skin was sutured and only the electrical connector of the smart catheter was left outside. The in vivo measurements were carried out one day after the smart catheter implantation. The MCAO surgery was performed according to the previous studies (32). A $4 / 0$ nylon filament covered with poly-Llysine was inserted through a puncture to the external carotid artery onto the common carotid artery. Multiple signals from the smart catheter were recorded once after MCA occlusion. Mean \pm SD values were calculated for all the measured parameters.

\section{RESULTS}

Table 1 lists the important specifications for each of the developed microsensors on the smart catheter. The performances of the microsensors were compared with commercial devices in vitro for each of the different measures.

Spontaneous waves of depolarization appeared in the ischemic penumbra during the third hour after MCA occlusion. As shown in Figure 2, the first episode of spreading depression (SD) consisting of negative DC deflections followed by positive waves $(3.8 \pm 0.6 \mathrm{mV})$ appeared $210 \pm 13 \mathrm{~s}$ after the occlusion. After a delay of $183 \pm 11 \mathrm{~s}$ from the onset of the negative DC shift the smart catheter recorded increased brain glutamate $(7.9 \pm 3.1 \mu \mathrm{mol} / \mathrm{L})$, decreased glucose $(21 \pm 11 \mu \mathrm{mol} / \mathrm{L})$, decreased and oxygen $(1.9 \pm 1.2 \mathrm{mmHg})$, and increased lactate $(24 \pm 17 \mu \mathrm{mol} / \mathrm{L})$. After that, intracranial pressure $(1.1 \pm 0.3 \mathrm{mmHg})$ was increased and brain temperature $\left(0.21 \pm 0.06^{\circ} \mathrm{C}\right)$ was increased.

\section{CONCLUSION}

Using a single device, we are able to record real-time changes in cerebral glucose, lactate, glutamate, oxygen, temperature, local cerebral blood flow and intracranial pressure. A system to correlate these variables to spreading depression has been established. These results demonstrate that the smart catheter can conduct the simultaneous and continuous measurement on multiple brain variables, within the ranges commonly observed in brain injury. Results further show the im- 


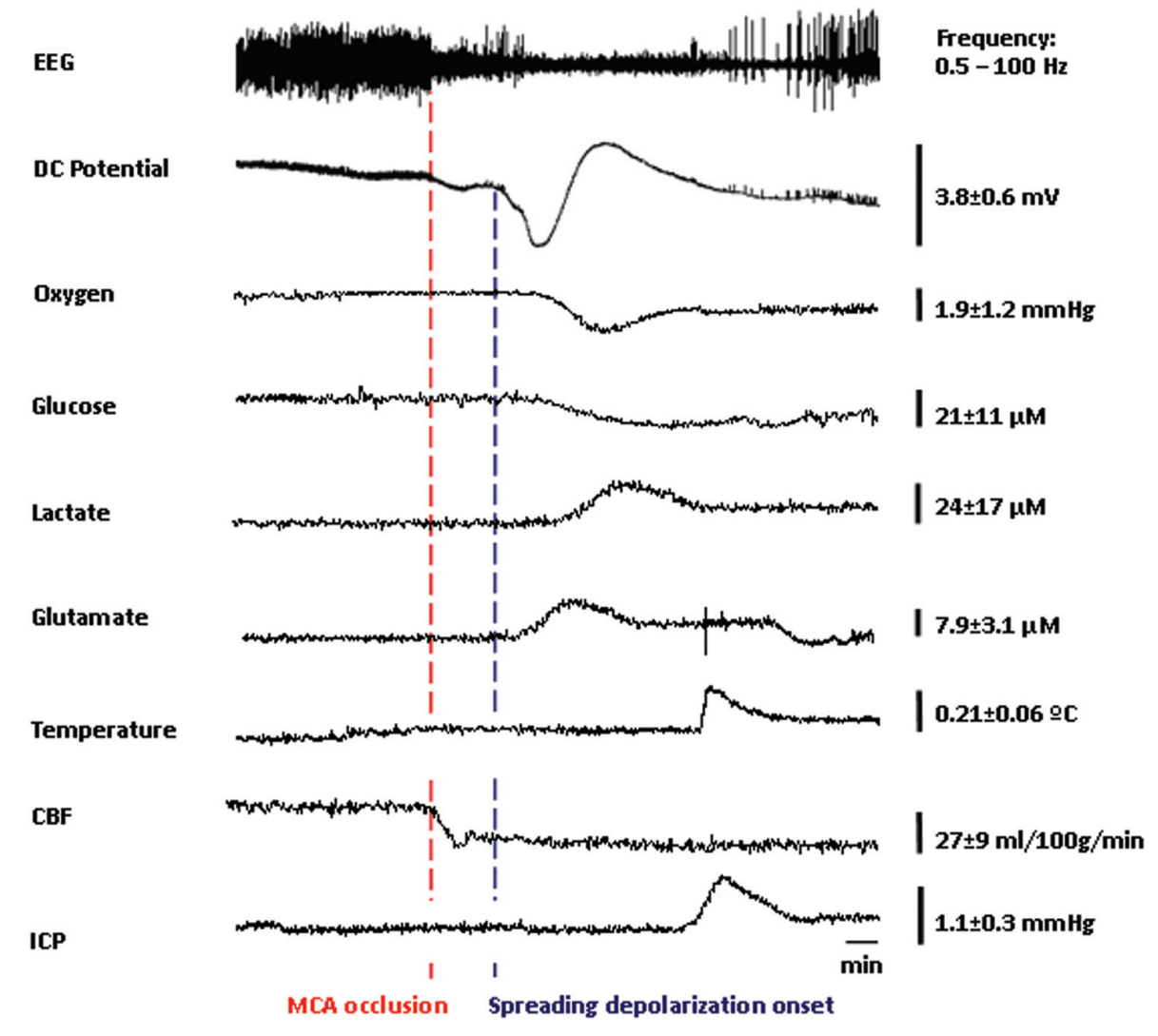

Figure 2. In vivo evaluation of smart catheter under permanent middle cerebral artery occlusion (MCAO) model: Experimental results after the first episode of spreading depression (SD) appeared after occlusion.

portance of multimodal measures of the same tissue to discover cause-effect relationships of candidate injury mechanisms. In that sense, the smart catheter represents a significant advancement in the field of neuromonitoring, opening up a completely new era in which medical decisions will be made based on extensive, real-time measures of brain chemistry and physiology during the critical period immediately following a brain injury, when the brain is most vulnerable to secondary insults. This advanced device not only can help with the clinical monitoring of brain-injured patients, but also serve a very valuable research device in the laboratory.

\section{ACKNOWLEDGMENTS}

This work was supported by the US Department of Defense under project No. PT090526P4(W81XWH-10-1-0978).

\section{DISCLOSURE}

The authors declare that they have no competing interests as defined by Bioelectronic Medicine, or other interests that might be perceived to influence the results and discussion reported in this paper.

\section{REFERENCES}

1. Menon DK, Schwab K, Wright DW, Mass AI, on behalf of The Demographics and Clinical Assessment Working Group of the International and Interagency Initiative toward Common Data Elements for Research on Traumatic Brain Injury and Psychological Health. (2010) Position statement: definition of traumatic brain injury. Arch. Phys. Med. Rehabil. 91:1637-40.

2. Coronado VG, et al. (2011) Surveillance for traumatic brain injury-related deaths-United States, 1997-2007. MMWR Surveill. Summary. 60:1-32.

3. Greve MW, Zink BJ. (2009) Pathophysiology of

4. Parikh S, Koch M, Narayan RK. (2007) Traumatic brain injury. Int. Anesthesiol. Clin. 45:119-35.

5. O'Connor WT, Smyth A, Cilchrist MD. (2011) traumatic brain injury. Mt. Sinai J. Med. 76:97-104.
Animal models of traumatic brain injury: a critical evaluation. Pharmacol. Ther. 130:106-13.

6. Narayan RK, et al. (2002) Clinical trials in head injury. J. Neurotrauma. 19:503-57.

7. Fakhry SM, Trask AL, Waller MA, Watts DD, IRTC Neurotrauma Task Force. (2004) Management of brain-injured patients by an evidencebased medicine protocol improves outcomes and decreases hospital charges. J. Trauma, 56:492-9.

8. Patel HC, Menon DK, Tebbs S, Hawker R, Hutchinson PJ, Kirkpatrick PJ. (2002) Specialist neurocritical care and outcome from head injury. Intensive Care Med. 28:547-53.

9. Cecil S, Chen PM, Callaway SE, Rowland SM, Adler DE, Chen JW. (2011) Traumatic brain injury: advanced multimodal neuromonitoring from theory to clinical practice. Crit. Care Nurse. 31:25-36.

10. Tisdall MM, Smith M. (2007) Multimodal monitoring in traumatic brain injury: current status and future directions. Br. J. Anaesth. 99:61-7.

11. Wartenberg KE, Schmidt JM, Mayer SA. (2007) Multimodality monitoring in neurocritical care. Crit. Care Clin. 23:507-38.

12. Stuart RM, et al. (2010) Intracranial multimodal monitoring for acute brain injury: a single institution review of current practices. Neurocrit. Care. 12:188-98.

13. Suarez JI. (2006) Outcome in neurocritical care: advances in monitoring and treatment and effect of a specialized neurocritical care team. Crit. Care Med., 34(SUPPL. 9):S232-8.

14. Messerer M, Daniel RT, Oddo M. (2012) Neuromonitoring after major neurosurgical procedures. Minerva Anestesiologica. 78:810-22.

15. Alves OL, Bullock R, Clausen T, Reinert M, Reeves TM. (2005) Concurrent monitoring of cerebral electrophysiology and metabolism after traumatic brain injury: an experimental and clinical study. J. Neurotrauma. 22:733-49.

16. Hajjhassan M, Chodavarapu V, Musallam S. (2008) NeuroMEMS: Neural probe microtechnologies. Sensors. 8:6704-26.

17. Grayson A. et al. (2004) A BioMEMS review: MEMS technology for physiologically integrated devices. Proc. IEEE. 92:6-21.

18. Song YA, Ibrahim AM, Rabie AN, Han J, Lin SJ. (2013) Microfabricated nerve-electrode interfaces in neural prosthetics and neural engineering. Biotechnol. Genet. Eng. Rev. 29:113-34.

19. Li C, Han J, Ahn CH. (2007) Flexible biosensors on spirally rolled micro tube for cardiovascular in vivo monitoring. Biosens. Bioelectron. 22:1988-93.

20. Li C, Wu PM, Han J, Ahn CH. (2008) A flexible polymer tube lab-chip integrated with microsensors for smart microcatheter. Biomed. Microdevices. 10:671-9.

21. Li C, Wu PM, Jung W, Ahn CH, Shutter LA, Narayan RK. (2009) A novel lab-on-a-tube for multimodality neuromonitoring of patients with traumatic brain injury (TBI). Lab Chip. 9:1988-90.

22. Tseng $\mathrm{IH}$, Chang CJ, Chang CW, Lu HH, Tsai MH. (2013) Effect of magnetron sputtered silicon nitride on the water-vapor-permeation-rate of polyimide thin film. Surf Coat Tech. 231:496-500. 
23. Wu Z, Li C, Bhattacharjee N, Hartings JA, Narayan RK, Ahn CH. (2013) A new intracranial pressure sensor on polyimide lab-on-a-tube using exchanged polysilicon piezoresistors. In: Solid-State Sensors, Actuators and Microsystems (TRANSDUCERS \& EUROSENSORS XXVII), 2013 Transducers $\&$ Eurosensors XXVII: The 17th International Conference on; 2013 Jun 16-20; Barcelona, Spain [Internet]. p 1779-82. [cited 2014 Nov 13]. Available from: http://ieeexplore.ieee.org/xpl/articleDetails.jsp? arnumber $=6627133 \&$ searchWithin\%3Da+new + intracranial\%26sortType\%3Dasc_p_Sequence $\% 26$ filter\%3DAND\%28p_IS_Number\%3A6626676\%29

24. Li C, Ahn CH, Shutter LA, Narayan RK. (2009) Toward real-time continuous brain glucose and oxygen monitoring with a smart catheter. Biosens. Bioelectron. 25:173-8.

25. Fontes MBA. (2013) Electrodes for bio-application: recording and stimulation. J. Physics Conf. Ser. 421:1-7.

26. Li C, et al. (2012) Brain-friendly amperometric enzyme biosensor based on encapsulated oxygen generating biomaterial. Conf. Proc. IEEE Eng. Med. Biol. Soc. 2012:6003-6.

27. Li C, et al. (2012) Micromachined lab-on-a-tube sensors for simultaneous brain temperature and cerebral blood flow measurements, Biomed. Microdevices. 14:759-6.

28. Li C, et al. (2012) Brain temperature measurement: A study of in vitro accuracy and stability of smart catheter temperature sensors. Biomed. Microdevices. 14:109-18.

29. Li C, et al. (2011) Smart catheter flow sensor for real-time continuous regional cerebral blood flow monitoring, Appl. Phys. Lett. 99:233705.

30. Menshykau D, Javier del Campo F, Munoz FX, Compton RG. (2009) Current collection efficiency of micro- and nano-ring-recessed disk electrodes and of arrays of these electrodes. Sens. Actuators B. Chem. 138:362-7.

31. Schmid-Elsaesser R, Zausinger S, Hungerhuber E, Baethmann A, Reulen HJ. (1998) A critical reevaluation of the intraluminal thread model of focal cerebral ischemia: evidence of inadvertent premature reperfusion and subarachnoid hemorrhage in rats by laser-Doppler flowmetry. Stroke. 29:2162-70.

32. Longa EZ, Weinstein PR, Carlson S, Cummins R. (1989) Reversible middle cerebral artery occlusion without craniectomy in rats. Stroke. 20:84-91.

Cite this article as: Li C, Narayan RK. (2014) Monitoring the injured brain. Bioelectron. Med. 1:4-8. 